This item was submitted to Loughborough's Research Repository by the author.

Items in Figshare are protected by copyright, with all rights reserved, unless otherwise indicated.

\title{
Influence of viscoelastic properties of encapsulation materials on the thermomechanical behavior of photovoltaic modules
}

PLEASE CITE THE PUBLISHED VERSION

https://doi.org/10.1109/JPHOTOV.2017.2762583

\section{PUBLISHER}

Institute of Electrical and Electronics Engineers

VERSION

AM (Accepted Manuscript)

\section{PUBLISHER STATEMENT}

Personal use of this material is permitted. Permission from IEEE must be obtained for all other uses, in any current or future media, including reprinting/republishing this material for advertising or promotional purposes, creating new collective works, for resale or redistribution to servers or lists, or reuse of any copyrighted component of this work in other works.

\section{LICENCE}

\section{All Rights Reserved}

\section{REPOSITORY RECORD}

Owen-Bellini, Michael, Daniel Montiel-Chicharro, Jiang Zhu, Tom Betts, and Ralph Gottschalg. 2019. "Influence of Viscoelastic Properties of Encapsulation Materials on the Thermomechanical Behavior of Photovoltaic Modules". figshare. https://hdl.handle.net/2134/27476. 


\title{
Influence of Viscoelastic Properties of Encapsulation Materials on the Thermomechanical Behavior of Photovoltaic Modules
}

\author{
Michael Owen-Bellini, Daniel Montiel-Chicharro, Jiang Zhu, Thomas R. Betts, Ralph Gottschalg \\ Centre for Renewable Energy Systems Technology, Wolfson School of Mechanical, Electrical and \\ Manufacturing Engineering, Loughborough University, United Kingdom, LE11 3TU
}

\begin{abstract}
In this work, the mechanical properties of encapsulation materials for photovoltaic (PV) modules have been studied. A finite-element (FEM) model has been developed to simulate the degradation of solder bonds within modules subjected to different environmental conditions. Various polymeric encapsulants are characterized using constitutive techniques and included in the model. It is shown that the degradation rates of the solder bonds is dependent on the behavior of the encapsulant and that some encapsulants may cause higher or lower degradation than others depending on the use-environment.
\end{abstract}

Index Terms - Polymer characterization; Photovoltaic Modules; Encapsulation; Durability; Solder bond fatigue; Viscoelasticity; Finite-Element;

\section{INTRODUCTION}

$\mathrm{D}$ URABILITY and lifetime of photovoltaic (PV) modules is one of the chief concerns for the industry, which is rapidly approaching maturity. Solder bond degradation is amongst the most dominant failure mechanisms for performance degradation and failure, particularly in hotter climates [1] and can be attributed to the thermomechanical stresses and strains generated during operation. The rate of degradation is known to be dependent on the specific use-environment, module design and material selection [2].

The encapsulant is an important component which provides mechanical stability and protection for the cells and interconnecting circuitry. Many encapsulation materials are available on the market today including Ethelyne Vinyl Acetate (EVA), Polyvinyl Butyryl (PVB), various Polyolefins (PL) and more recently Ionomers. These materials are all complex polymer thermosets whose mechanical behavior is heavily dependent on both temperature and rate of stress application. It is shown here that the variable nature of different environments [3] influence the mechanical behavior of the encapsulants in different ways and that this subsequently effects the degradation potential of solder bonds.

In this work, the mechanical behavior of 5 types of encapsulants are examined. The materials include two types of EVA (EVA-1 and EVA-2), a PL, a PVB and an Ionomer. First, the viscoelastic properties of each encapsulant are characterized through experimental and analytical means. A finite element model (FEM) of silicon wafer-based PV minimodules is then developed to simulate the thermomechanical stresses for temperature histories from 2 different climate zones as well as the thermal cycling module certification protocol, TC200 [4]. The potential for solder bond degradation is examined through calculation of the viscoplastic deformation.

\section{MODELING VISCOELASTICITY}

Viscoelasticity is a property which describes materials which demonstrate both elastic and viscous behavior characteristics. For an applied stress, the corresponding strain component is a function of both temperature and time. The stress response to an applied strain experiences greater delay than at lower temperatures, where the material is more elastic. It is a complex property which requires an equally complex constitutive model to adequately characterize.

\section{A. Generalized Maxwell Model}

The behavior of a simple linear viscoelastic material can be represented as a spring-dashpot combination. Where the spring and dashpot represent the elastic and viscous portions of the material, respectively. This model can be expanded to adequately capture the behavior of more complex polymers such as those used for PV module encapsulation. The Generalized Maxwell model utilizes multiple spring-dashpots, hereby referred to as Maxwell elements, in a parallel configuration (Fig. 1). Each Maxwell element has its own properties which define its behavior. The number of elements required is dependent on the complexity of the material. The model may be expressed mathematically as (1).

$$
G^{*}(\omega)=G_{\infty}+\sum_{n=1}^{m} G_{n} \frac{\omega \tau_{n}}{1+\omega \tau_{n}}
$$

Where $G^{*}(\omega)$ is the complex modulus, $\mathrm{G}_{\infty}$ the instantaneous modulus, $m$ the number of Maxwell elements, $\omega$ the frequency of the applied strain, $G_{n}$ and $\tau_{n}$ the relaxation strength and relaxation time for the $\mathrm{n}^{\text {th }}$ Maxwell element, respectively. The Maxwell element parameters can be determined experimentally and analytically, as described in the following subsection. The number of Maxwell elements required for accurate modelling is determined by the complexity of the material. 


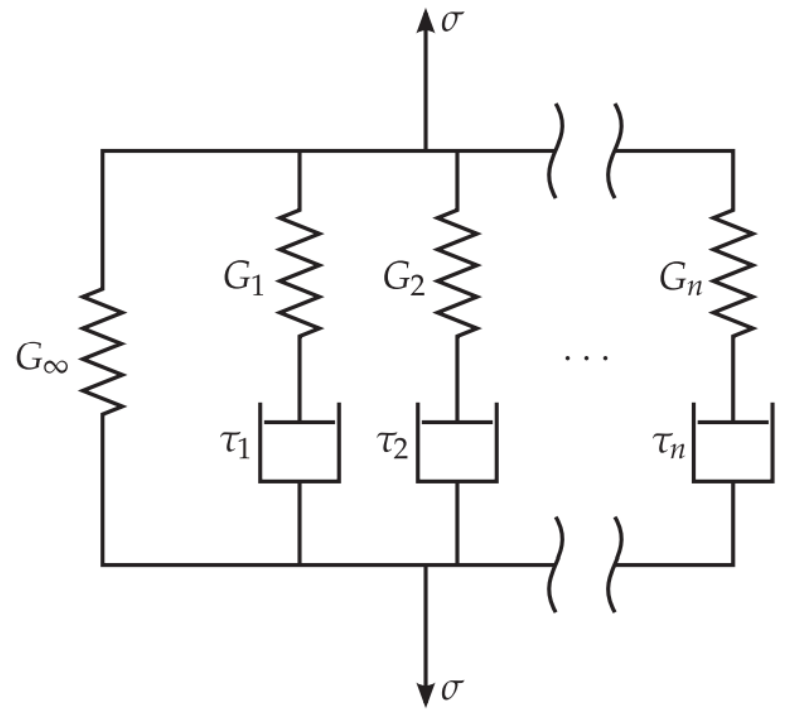

Fig. 1: Graphical representation of the Generalized Maxwell Model with an applied stress, $\sigma$.

\section{B. Identifying Maxwell Terms}

Accurate modeling of viscoelastic behavior requires appropriate determination of the number of Maxwell elements and the parameter values, $G_{n}$ and $\tau_{n}$, for each element. The storage modulus of a material measured over an extended range of frequencies can be used to determine the Maxwell parameters. In this work, the storage modulus of each material has been measured using a TA Instruments Dynamic Hybrid Rotational Rheometer (DHR-2). The rheometer applies an oscillatory strain to disc-shaped samples of the materials with $8 \mathrm{~mm}$ diameter and $0.8 \mathrm{~mm}$ thickness. Each material has been cured according to the manufacturers recommended specifications. The storage modulus may be determined by measuring the stress response. To characterize the material as accurately as possible, the storage modulus should be measured over a wide range of oscillating frequencies (typically $10^{-14}$ $10^{14} \mathrm{~Hz}$ ). Such measurements are quite impractical and to overcome this, an alternative analytical approach is employed. The storage modulus is measured in the range of 0.1 to $10 \mathrm{~Hz}$ at multiple isothermal conditions ranging from $-40^{\circ} \mathrm{C}$ to $150^{\circ} \mathrm{C}$ in steps in $10^{\circ} \mathrm{C}$. The Time-Temperature Superposition Principle (TTSP), which states that increasing frequency at a constant temperature or increasing temperature at a constant frequency are equivalent, may be used to shift the isotherms such that a single mastercurve may be constructed. The mastercurve covers a much wider range of frequencies as required. Williams-Landel-Ferry (WLF) [5] outlined an empirical equation (2) to describe the quality of a TTSP shift.

$$
a_{T}=-\frac{C_{1}\left(T-T_{r e f}\right)}{C_{2}+\left(T-T_{\text {ref }}\right)}
$$

Where $\alpha_{\mathrm{T}}$ is the shift coefficient, $\mathrm{C}_{1}$ and $\mathrm{C}_{2}$ are material dependent constants and the reference temperature, $\mathrm{T}_{\text {ref. }}$. Isothermal measurements for EVA-1 are shown in Fig. 2. A gradual increase in storage modulus is observed with decreasing temperature, with a rapid increase in storage modulus as temperatures reach those of the glass transition region (approx. $-30^{\circ} \mathrm{C}$ ). Conversely, with increasing temperature a decrease in storage modulus can be observed until a complete melting of the material is reached at around $100^{\circ} \mathrm{C}$

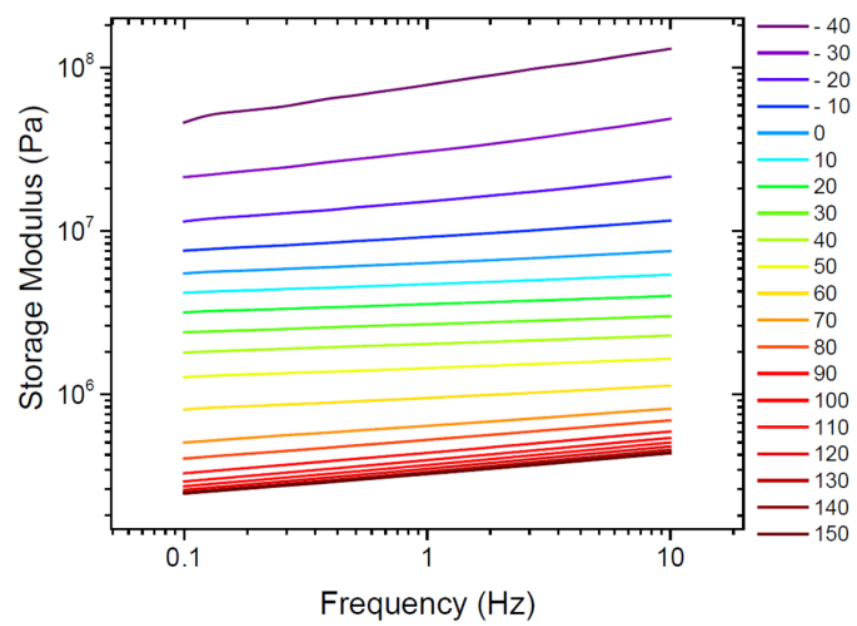

Fig. 2: Storage modulus measurements for EVA-1 at various isotherms

A mastercurve has been produced for each material at a reference temperature of $20^{\circ} \mathrm{C}$ as shown in Fig.3. The choice of reference temperature is arbitrary, and has no discernable impact on the quality of the shift. The WLF parameters and fit quality are outlined in Table I.

Table I: WLF shifting parameters

\begin{tabular}{cccc}
\hline \hline Encapsulant & $\mathrm{C} 1$ & $\mathrm{C} 2(\mathrm{~K})$ & $\mathrm{R}^{2}$ \\
\hline \hline EVA-1 & 41.32 & 242.12 & 0.98 \\
EVA-2 & 38.22 & 238.06 & 0.97 \\
Ionomer & 69.17 & 606.69 & 0.99 \\
PL & 58.61 & 382.22 & 0.98 \\
PVB & 22.57 & 242.69 & 0.99 \\
\hline \hline
\end{tabular}

Both EVA types, PL and Ionomer exhibit similar mastercurves, particularly in the region between $10^{-10}-10^{-2}$ $\mathrm{rad} / \mathrm{s}$. Greater disparities between these encapsulants emerge outside of this range, especially with the ionomer which demonstrates a sharp reduction in storage modulus at the lower frequencies. PVB demonstrates an entirely different mastercurve, undergoing a rapid change between $10^{-3}$ and $10^{3}$ $\mathrm{rad} / \mathrm{s}$. This change corresponds with the rapid material change which can be observed between $10^{\circ} \mathrm{C}$ and $50^{\circ} \mathrm{C}$, where the material experiences a dramatic loss in stiffness. It is quite 
common for modules to operate within this range and so the stability of the material could be quite important here. All other materials exhibit a greater level of stability in this range, where the storage modulus does not change as significantly.

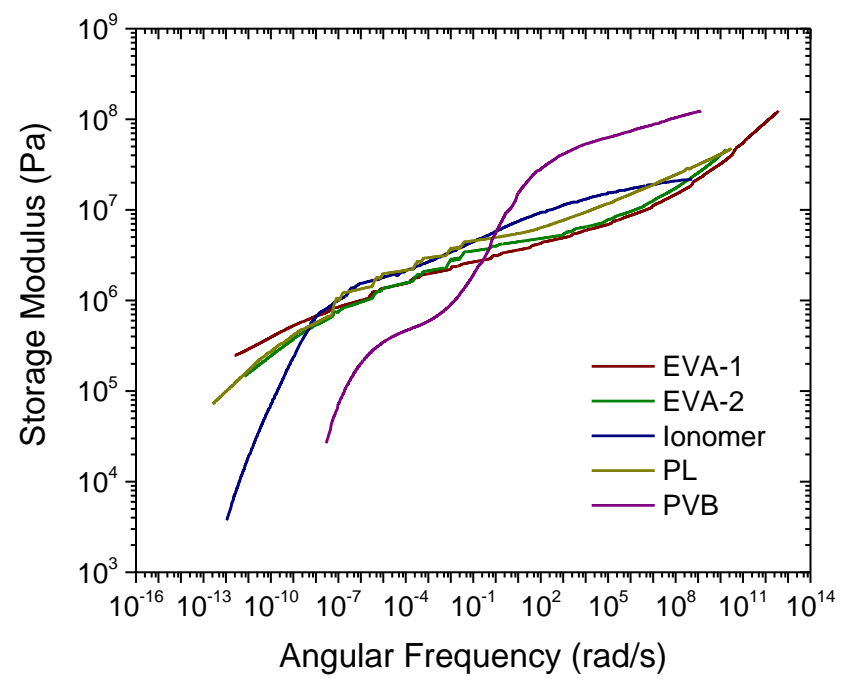

Fig.3: Mastercurves for each material with a reference temperature of $20^{\circ} \mathrm{C}$

A polynomial least-squared fitting algorithm is used to fit the Generalised Maxwell Model equation (1) to the mastercurves, such that the optimum number of elements and the corresponding values can be determined. The Maxwell elements are shown in Fig. 4 for each material. It was found that 28 elements gave the most acceptable fit ensuring an $\mathrm{R}^{2}$ of at least 0.98 for each material whilst also allowing for acceptable computation times for the subsequent FEM simulations.

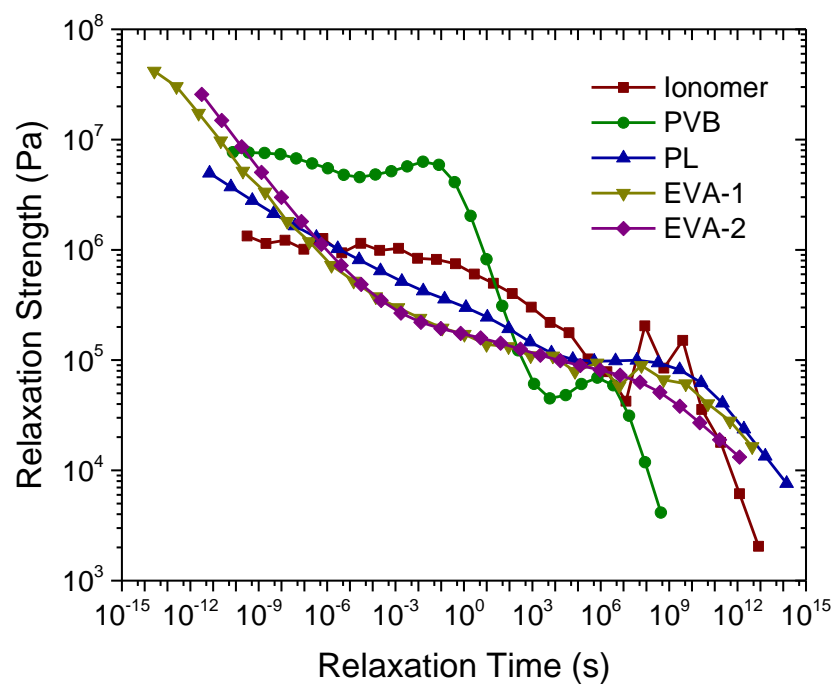

Fig. 4 : Fitted Maxwell terms for each material

\section{Model Validation}

Stress relaxation is characteristic of viscoelastic behavior. Following the application of a step deformation, the stress (or force) necessary to maintain the deformation decays with time. The stress relaxation of the materials is used to validate the viscoelastic model parameters. A step deformation is applied to the samples with an application time of $0.01 \mathrm{~s}$ at constant temperatures of $-40,-20,0$ and $80^{\circ} \mathrm{C}$. The resulting stress is monitored over a period of 10 minutes. The experiment is modeled in an FEM software package and simulated using the Maxwell element parameters as inputs. Fig.5 demonstrates the stress relaxation experiment and simulation for EVA-1 at $0^{\circ} \mathrm{C}$ and $80^{\circ} \mathrm{C}$. As anticipated for this material, stress relaxation is greater at $80^{\circ} \mathrm{C}$ where the storage modulus is lower. A good agreement between the experimental and simulation data is achieved for all materials and temperatures, where the ionomer had the worst agreement with a root mean biased error of 0.07 at $-40^{\circ} \mathrm{C}$.

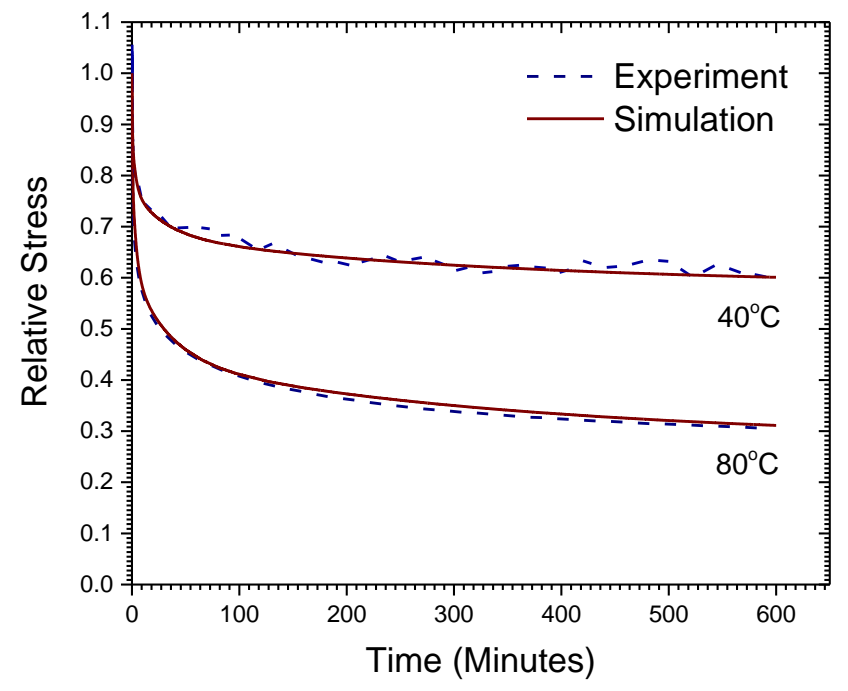

Fig.5: Experimental and simulated stress relaxation for EVA-1 at $80^{\circ} \mathrm{C}$ and $0^{\circ} \mathrm{C}$

\section{FINITE-ELEMENT MODEL}

Direct measurements of the thermomechanical strains generated during thermal loading presents a difficult challenge due to the complex nature of PV module assemblies. Instead, an FEM approach is employed to estimate the stresses and strains generated, with a focus on the thermomechanical stresses at the solder bonds. A 2D model has been developed using COMSOL Multiphysics which reflects silicon waferbased mini-modules fabricated at Loughborough University.

\section{A. Model Geometry}

The mini-modules produced at Loughborough consist of 6 monocrystalline silicon full-square cells soldered in series using a $60 \mathrm{Sn} 40 \mathrm{~Pb}$ solder alloy and copper-based 
interconnecting ribbons. These are then laminated with a float glass front cover, polymeric backsheet and encapsulant. Table II summarizes the dimensions of each component.

Table II: Dimensions for the components in the model, $\mathrm{mm}$

\begin{tabular}{cccc}
\hline \hline Component & Length & Width & Height \\
\hline \hline Cell & 156 & 156 & 0.22 \\
Glass & 538 & 359 & 2.9 \\
Encapsulant & 538 & 359 & 0.8 \\
Backsheet & 538 & 359 & 0.41 \\
Ribbons & $156-318$ & 1.5 & 0.3 \\
Solder & 156 & 1.5 & 0.02 \\
\hline \hline
\end{tabular}

A screen capture of the finite-element model is presented in Fig. 6, showing a cross sectional view of the model focused at the edge of the outer cell displaying the ribbon which is connected to the adjacent, inner cell. Whilst the copper-ribbons are solder-coated, the outer layer of solder is considered to have a negligible impact on the behavior of the device and has therefore been ignored in this work in the interest of simplification of the model and a reduction in computational requirements.

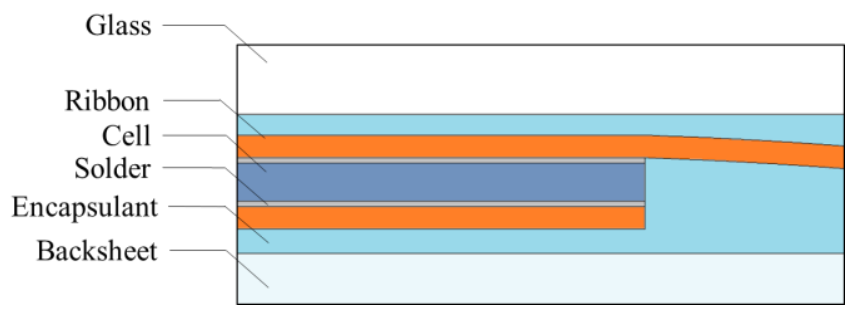

Fig. 6: Schematic of part of the 2D FEM geometry

\section{B. Material Properties}

Appropriate material properties must be defined in the model in order to produce appropriate results. Besides the encapsulant, all materials are considered to be linearly elastic. The relevant material properties have been summarized in Table III.

Table III: Material properties used in the model

\begin{tabular}{ccccc}
\hline \hline Component & $\begin{array}{c}\text { CTE }\left(10^{-}\right. \\
6 / \mathrm{K})\end{array}$ & $\begin{array}{c}\text { Young's } \\
\text { Modulus } \\
(\mathrm{GPa})\end{array}$ & $\begin{array}{c}\text { Poisson's } \\
\text { Ratio }\end{array}$ & $\begin{array}{c}\text { Density } \\
\left(\mathrm{g} / \mathrm{cm}^{3}\right)\end{array}$ \\
\hline \hline Cell & 2.7 & 128 & 0.28 & 2.3 \\
Glass & 8 & 73 & 0.23 & 2.5 \\
Backsheet & 5.04 & 3.5 & 0.29 & 2.5 \\
Ribbons & 17 & 105 & 0.35 & 8.5 \\
Solder & 21 & 4.2 & 0.4 & 8.4 \\
\hline \hline
\end{tabular}

Creep behavior and plastic deformation of the solder bonds is considered in this work as a means of evaluating damage potential. Anands viscoplastic model [6] is employed using parameters which have been well-defined in the literature for eutectic $60 \mathrm{Sn} 40 \mathrm{~Pb}$ solder [7].

\section{THERMO-MECHANICAL BEHAVIOR}

\section{A. Module Temperature Data}

Thermomechanical behavior is simulated through module temperatures according to the thermal cycling 200 program outlined in IEC61215 and for 2 outdoor climates, one hot and one cold. Table IV outlines the locations used. Data for Gobabeb is obtained from the baseline surface radiation network (BSRN), whereas data from Loughborough are measurements taken on-site. For both sites, module temperatures are estimated from ambient temperature and global horizontal irradiance using the thermal model outlined by Fuentes [8] and the in-plane irradiance model by Perez et al [9]. For Gobabeb, one day is taken at the height of summer where temperatures are highest and for Loughborough one day is taken in the winter where temperatures are lowest. As such, the performance of each encapsulant can be evaluated for highest and lowest temperatures.

Table IV: Locations used for simulations

\begin{tabular}{ccc}
\hline \hline Location & Climate Type & Latitude $\left(^{\circ}\right)$ \\
\hline \hline Gobabeb, Namibia & Desert & -23.56 \\
Loughborough, UK & Temperate & 52.77 \\
\hline \hline
\end{tabular}

\section{B. Solder Bond Degradation Potential}

Energy dissipation density is a measure of the accumulated inelastic (or non-recoverable) strain energy which is lost through creep and plastic deformation. It is commonly used as a metric for fatigue studies of metals and plastics. In this work the energy dissipation density of the solder is calculated as a means of evaluating the damage potential of the solder bonds within the PV module assembly. The rate of energy dissipation at the high temperature dwell of TC200 is plotted for the top and bottom solder bonds in the middle cell in Fig.7. It is found that the bottom bond undergoes a higher rate of accumulation, likely due to the additional strains imposed through the interconnecting ribbon following displacement of the solar cells. The energy dissipation density is therefore only reported for the bottom solder bond for all subsequent simulations. 


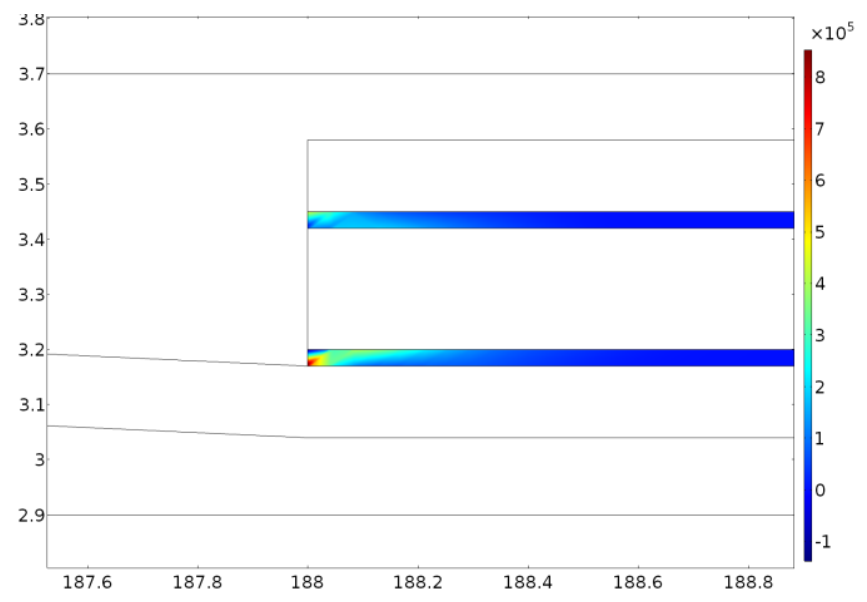

Fig.7: Screen capture of the rate of creep energy dissipation density $\left(\mathrm{J} / \mathrm{m}^{3}\right)$ at the high temperature dwell during thermal cycling

The energy dissipation density is calculated for each encapsulant through each thermal profile. Energy dissipation density for each encapsulant following the TC200 thermal profile is presented in Fig.8. At the low temperature dwell (around 2500s), both EVA encapsulants appear to have no discernable difference, sharing the highest amount of dissipated energy. The ionomer exhibits the lowest amount of dissipated energy. A total change in temperature of $125^{\circ} \mathrm{C}$ occurs during the ramp up from the low temperature dwell to the high temperature dwell. This has a significant effect on the inelastic deformation of the solder bond, and is the period in which the majority of deformation and damage occurs. Whilst the melting point of the solder alloy is not reached, higher temperatures increase the rate of creep and viscoplastic deformation. At the high temperature dwell (8000s), EVA-2 appears to have accumulated the most damage whilst the ionomer has accumulated far less than the other encapsulants, with approximately $12 \%$ less energy dissipation than the next encapsulant, PL. Both PL and PVB appear to have dissipated the same amount of energy.

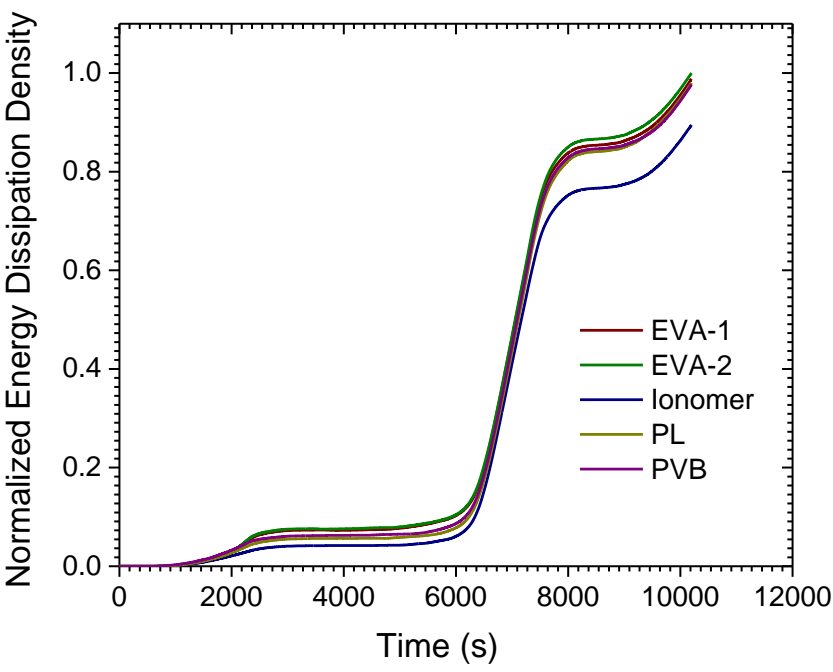

Fig.8: Calculated energy dissipation density for each encapsulant following thermal cycling 200

It might be expected that, based on these results, the ionomer would be the best performing encapsulant, with EVA2 being the worst performing in terms of damage to the solder bonds. However, the thermal profile used for the TC200 tests are not representative of temperature conditions experienced by modules in real environments. Such a rapid increase in temperature over an extended period would not occur. Given the time-dependent nature of the encapsulants mechanical behavior, it is important to look at more realistic conditions. Under different rates of temperature change, the encapsulants could behave differently, affecting the viscoplastic deformation potential in a much different way. For this reason, the energy dissipation density is calculated for the 2 climates outlined previously. Energy dissipation density for the colder climate, Loughborough, is presented in Fig.9. In this climate, a maximum temperature change of $18^{\circ} \mathrm{C}$ occurs over a period of $\sim 4$ hours from $0^{\circ} \mathrm{C}$ to $18^{\circ} \mathrm{C}$. Both the rate of change of temperature and the absolute temperatures are significantly lower than the TC200 profile. As would be expected, the total energy dissipated is significantly lower. The greatest deformation occurs during the increase in temperature during sunrise. Whilst it appears as though no deformation occurs following peak temperature (50000s), a slight gradual change does occur, though deformation during cooling at such low temperatures are marginal.

Contrary to the results presented in Fig.8, EVA-2 is amongst the lowest in terms of energy dissipated, having dissipated 5.4\% less energy than EVA-1. This would suggest that EVA-2 is more stable within this temperature range. The ionomer continues to be the best performer for this climate, and there is a more significant difference between the other encapsulants. If conclusions were to be drawn based solely on the certification testing procedures, then EVA-2 might be considered the worst encapsulant. However, in the field under realistic operating conditions, it might be that EVA-2 is one of the better performers. In addition to this, despite both EVAs 
being based on the same material, it would appear that the additives and processing introduced by the manufacturer leads to a marked difference in mechanical behavior. It should be noted here that the materials being studied have been designed as "fast cure" and "ultra-fast cure", for EVA-1 and EVA-2, respectively. This refers to the recommended time required for lamination.

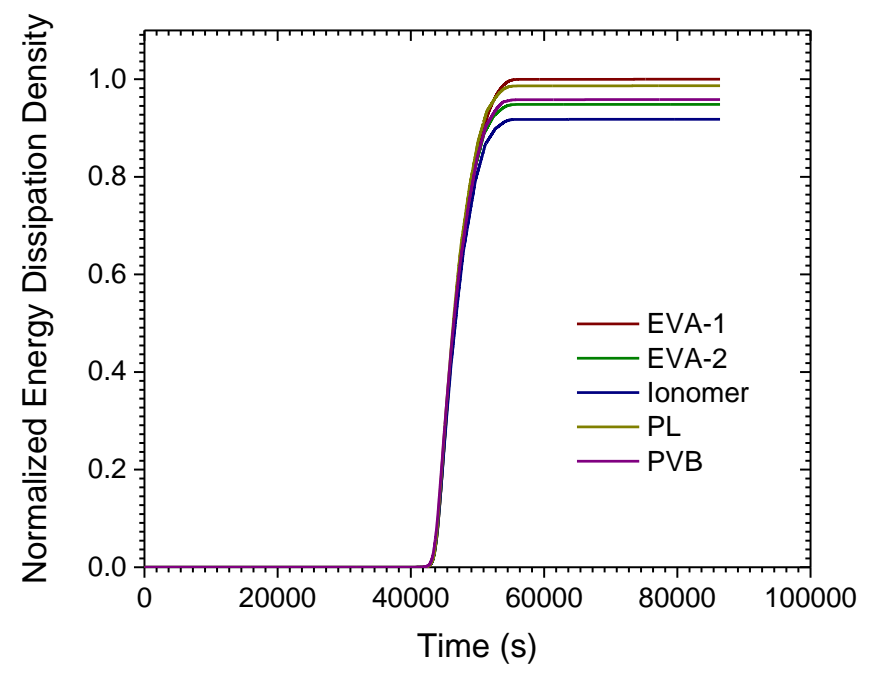

Fig.9: Energy dissipation density for the cold environment, Loughborough

Lastly, energy dissipation density for the hot location, Gobabeb, is presented in Fig.10. In this case, a total temperature change of $50^{\circ} \mathrm{C}$ occurs over a time period of approximately 6 hours from $18^{\circ} \mathrm{C}$ to $68^{\circ} \mathrm{C}$. The elevated temperature and duration of the temperature rise has resulted in a significantly higher energy dissipation than seen in the cold climate. At the peak temperature ( $46000 \mathrm{~s})$ it is found that the PVB results in the greatest amount of energy dissipation. Interestingly, EVA-1 becomes the better performing EVAbased encapsulant for this environment. A similar difference is demonstrated between EVA-1 and PL. The ionomer is once again the encapsulant which causes the least energy dissipation.

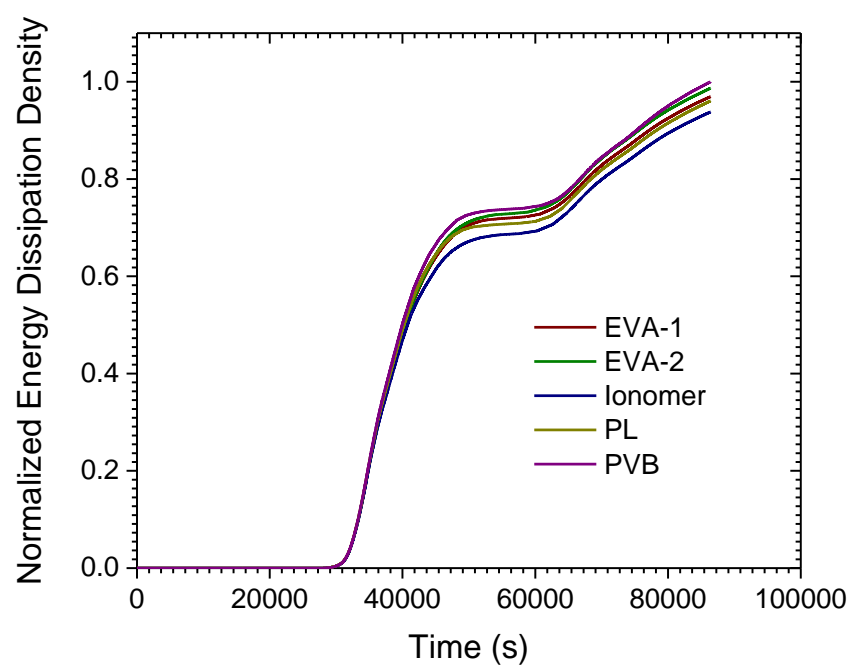

Fig.10: Energy dissipation density for hot environment, Gobabeb

\section{CONCLUSIONS}

Degradation of solder bonds within a PV module assembly was evaluated through simulation of the viscoplastic deformation under 3 environmental conditions and quantified through calculation of the energy dissipation density. Module operating temperatures for real outdoor environments, one cold and one hot, were used for the simulations as well as the thermal cycling profile mandated by the IEC61215 certification protocols. The viscoelastic properties of the encapsulation materials has been shown to have a direct influence on the imposed strains (and therefore degradation potential) of the solder bonds. The mechanical behavior for each encapsulant is dependent on the absolute temperature and the rate of change of temperature, as expected of the viscoelastic properties.

The ionomer-based encapsulant was found be the best performer, with the lowest calculated total energy dissipation under each condition. This is likely due to the higher physical stability demonstrated by the encapsulant, which does not undergo dramatic material phase change with temperature compared with the other encapsulants. The other encapsulation materials have been shown to perform better or worse than each other, depending on the climate. Whilst EVA-2 and PVB outperformed EVA-1 and PL in the colder environment, the opposite was the case for the hot environment. The environment-dependent performance is indicative of the viscoelastic properties of the encapsulants, where the elastic modulus is dependent on the absolute temperature and the rate of strain which is applied during changes in temperature.

In addition to this, the certification testing protocol TC200 suggested that the degradation potential of solder bonds would be highest when EVA-2 was used as the encapsulation material, and lowest when ionomer was used with no discernable difference between the other encapsulants. To 
draw any conclusions about the ability of an encapsulant to provide mechanical stability based on these tests could be misleading, as the encapsulant may perform better or worse depending on the location of deployment.

\section{ACKNOWLEDGMENTS}

Acknowledgments are made to the BSRN for supplying climate data used in the analysis and to Prof. Philip Eames for providing access to the rheometer. This work is funded by the STAPP (EP/H040331/1) and JUICE (EP/P003605/1) projects.

\section{REFERENCES}

[1] D. C. Jordan, T. J. Silverman, J. H. Wohlgemuth, S. R. Kurtz, and K. T. VanSant, "Photovoltaic failure and degradation modes," Prog. Photovoltaics Res. Appl., vol. 25, no. 4, pp. 318-326, Apr. 2017.

[2] D. C. Jordan, S. R. Kurtz, K. Vansant, and J. Newmiller, "Compendium of photovoltaic degradation rates," Prog. Photovoltaics Res. Appl., vol. 24, no. February 2016, pp. 978-989, 2016.

[3] M. Owen-bellini, J. Zhu, T. R. Betts, and R. Gottschalg, "Environmental Stress Potentials of Different Climatic Regions," in 31st European PV Solar Energy Conference and Exhibition, 2015, no. 0.

[4] International Electrotechnical Commision, "IEC 61215: Crystalline Silicon Terrestrial Photovoltaic (PV) ModulesDesign Qualification and Type Approval," International Electrotechnical Commission, vol. 3. 2005.

[5] M. L. Williams, R. F. Landel, and J. D. Ferry, "The Temperature Dependence of Relaxation Mechanisms in Amorphous Polymers and Other Glass-forming Liquids.," $J$. Am. Chem. Soc., vol. 77, no. 12, pp. 3701-3707, 1955.

[6] L. Anand, "Constitutive equations for hot-working of metals," Int. J. Plast., vol. 1, no. 3, pp. 213-231, 1985.

[7] Z. N. Cheng, G. Z. Wang, L. Chen, J. Wilde, and K. Becker, "Viscoplastic Anand model for solder alloys and its application," Solder. Surf. Mt. Technol., vol. 12, no. 2, pp. 31-36, 2000.

[8] M. K. Fuentes, “A Simplified Thermal Model for Flat-Plate Photovoltaic Arrays," Sandia Report. Sandia National Laboratories, 1987.

[9] R. Perez, P. Ineichen, R. Seals, J. Michalsky, and R. Stewart, "Modeling daylight availability and irradiance components from direct and global irradiance," Sol. Energy, vol. 44, no. 5, pp. 271-289, 1990. 